\title{
A Smartphone-based Sensing Platform to Model Aggressive Driving Behaviors
}

\author{
Jin-Hyuk Hong, Ben Margines, Anind K. Dey \\ Human-Computer Interaction Institute, Carnegie Mellon University \\ \{hjinh7, benjamin.margines\}@gmail.com, anind@cs.cmu.edu
}

\begin{abstract}
Driving aggressively increases the risk of accidents. Assessing a person's driving style is a useful way to guide aggressive drivers toward having safer driving behaviors. A number of studies have investigated driving style, but they often rely on the use of self-reports or simulators, which are not suitable for the real-time, continuous, automated assessment and feedback on the road. In order to understand and model aggressive driving style, we construct an invehicle sensing platform that uses a smartphone instead of using heavyweight, expensive systems. Utilizing additional cheap sensors, our sensing platform can collect useful information about vehicle movement, maneuvering and steering wheel movement. We use this data and apply machine learning to build a driver model that evaluates drivers' driving styles based on a number of driving-related features. From a naturalistic data collection from 22 drivers for 3 weeks, we analyzed the characteristics of drivers who have an aggressive driving style. Our model classified those drivers with an accuracy of $90.5 \%$ (violation-class) and $81 \%$ (questionnaire-class). We describe how, in future work, our model can be used to provide real-time feedback to drivers using only their current smartphone.
\end{abstract}

\section{Author Keywords}

Driving assessment, smartphone, in-vehicle sensing platform

\section{ACM Classification Keywords}

H.5.m. Information interfaces and presentation (e.g., HCI): Miscellaneous.

\section{INTRODUCTION}

Driving is an integral part of many peoples' lives, as it allows them to travel faster and further to accomplish more. It even provides for an improved quality of life [29]. However, it also exposes individuals to the risk of being involved in harmful and even fatal accidents [5, 8]. Not only are these accidents injurious to the people involved, but also they are expensive: accident-related costs in 2011

Permission to make digital or hard copies of all or part of this work for personal or classroom use is granted without fee provided that copies are not made or distributed for profit or commercial advantage and that copies bear this notice and the full citation on the first page. Copyrights for components of this work owned by others than ACM must be honored. Abstracting with credit is permitted. To copy otherwise, or republish, to post on servers or to redistribute to lists, requires prior specific permission and/or a fee. Request permissions from permissions@acm.org.

CHI 2014, April 26-May 1, 2014, Toronto, Ontario, Canada.

Copyright (CACM 978-1-4503-2473-1/14/04 ..\$15.00.

http://dx.doi.org/10.1145/2556288.2557321 in the US were estimated to be $\$ 299.5$ billion [6]. Road traffic accidents are directly attributable to human behavior because they are related to vehicle control and sensitive to the complex driving environment (e.g., weather, traffic) [7, 30]. Significant research has been performed to understand the relationship between human behavior and road safety, for example, asking which behavioral factors can indicate 'good' or 'bad' driving, or which behavioral factors might convey the risk that a driver poses to others $[3,18]$.

Aggressive driving, a particular type of driving style, has long been studied due to its strong correlation with accidents and traffic safety hazards: by one estimate, it was influential in causing the majority of accidents in the United States from 2003 to 2007. [1]. This style of driving refers to a behavior that deliberately or likely increases the risk of collision and is motivated by impatience, annoyance, hostility and/or an attempt to save time [35]. A similar definition by the NHTSA refers to aggressive driving as that which "directly affects other road users by placing them in unnecessary danger" [9]. More specifically, it may involve a variety of behaviors such as excessive speeding, tailgating, weaving in and out of traffic, and improper lane changes $[17,35]$. Aggressive driving is a major contributing factor to road accidents and is now perceived as being one of the most significant problems of modern day driving [33].

By understanding an individual's driving style and providing feedback, he or she may change driving styles to reduce aggressive driving. For example, a monitoring system may reduce aggressive driving through voluntary recommendations (e.g., safety belt use [28]) or involuntary controls (e.g., speed governor [15]). Drivers can also manage situational and environmental factors that may motivate or stimulate them to drive more aggressively. To support this feedback, we need an automated method for assessing driving style in real-time.

Many approaches have been used to understand and assess one's driving style, and in-vehicle sensing technology, which yields more objective measures of driving style, has shown its potential for real-time, continuous, automated assessment and feedback on the road [5, 18, 36]. From cameras to an OBD2 connector (on-board diagnostic system that collects variables from the car computer, such as engine RPM and vehicle speed), a number of in-vehicle sensing platforms have been developed to collect a variety of information on the driver, the vehicle and their driving environment [2, 14]. However, because the systems are 
typically cost thousands of dollars, most consumers are not able to afford to equip their cars, limiting the overall applicability to the general public. We are interested in a solution that is accessible to all drivers.

To meet this need, we propose to model aggressive driving by: 1) constructing a lightweight in-vehicle sensing platform based on drivers' own smartphones to provide an objective measurement of driving style, 2) using a machine learning (ML) technique with a number of driving-related features for an automated assessment of driving style, and 3 ) evaluating our approach with real-life daily driving data. In the future, such models can be used to identify aggressive driving in real time and to provide feedback to the driver to encourage a change in driving style.

Using our lightweight sensor platform, we collected naturalistic driving data from 22 participants for three weeks and were able to differentiate between aggressive and non-aggressive driving styles. To assess driving style, driver models are built using a machine learning technique and a number of sensor-based driving features. These models identify drivers who have an aggressive driving style with an accuracy of $90.5 \%$ (violation-class) and $81 \%$ (questionnaire-class). We discuss the limitations of our system, describe how it can support driving feedback, and identify opportunities for future work.

\section{ASSESSMENT OF DRIVING STYLE}

The ability to provide feedback to drivers about their driving style, especially if it is aggressive, can help drivers to become more aware of and improve their behavior. This would likely reduce the probability of poor driving outcomes. Because of this potential, a number of different assessment approaches have been investigated, including questionnaires [32], simulated driving environments [20], in-vehicle sensing technology [31], and expert-based examinations [26]. Figure 1 ranks these assessment methods along the dimensions of cost and how easily they can be applied to evaluations of real-world driving.

Relevant questionnaires have been created that ask about a driver's personality and driving styles to understand his risk of having an accident $[4,7,33,38]$. The questionnaires are simple and inexpensive to administer. Those targeted toward assessing aggressive driving include the Aggressive Driving Behavior Scale [17], the Driving Vengeance Questionnaire [37], the Driving Anger Scale [11], and the Driving Anger Expression Inventory [12]. These questionnaires have allowed for great progress in studying driving style and driver aggression [10]. Of these, the most popular questionnaire is the Manchester Driving Behavior Questionnaire (DBQ). The DBQ was initially developed to investigate whether a questionnaire could distinguish between driving errors and driving violations; the authors that three factors could explain 33\% of the variance in aggressive driving - violations, hazardous errors, and nonhazardous errors [32].

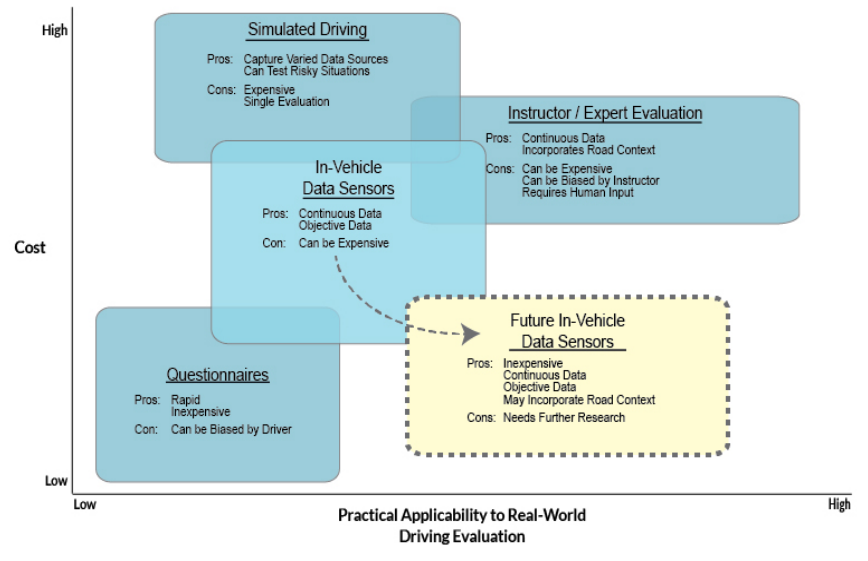

Figure 1. Ranking of existing assessment methods

Despite their clear benefits, questionnaires are not ideal when applied to the creation of an automated and objective assessment system. The very nature of the data collection method precludes real-time evaluation: it would be unsafe to ask drivers to take a questionnaire while driving. Furthermore, because questionnaires are answered post hoc, drivers need to rely on their memory, which can be flawed due to impression-management and self-deception. Consequently, self-reports may be biased and may not yield sufficient information to interpret vehicle driving, which is a complex task composed of continuous and reactive interactions between the driver, the vehicle and their environment $[5,34,36,40]$. Taken together, another approach to studying driving style is required to obtain objective, real-time data.

An alternative approach for assessing driving style is to use a simulated driving environment. Here, a driver controls a vehicle in a virtual driving environment, providing a safe and somewhat naturalistic way to study driver behavior. One key benefit of simulators is that they can be manipulated to put the drivers in situations which could be unsafe in a real driving environment, e.g., to simulate an accident or near-accident. Except for academic research or training purposes however, simulated driving environments are used less frequently to provide real-time driving feedback, as they can be quite expensive and risk misrepresenting reality.

In many countries, an expert-based examination is administered as an initial test to judge driving performance. Here, a certified driving expert evaluates and provides appropriate feedback in real time about a driver's skill and style. It has been widely adopted in road tests of driving ability. Because no other real-time alternatives exist, expert-based examination is used by default for licensing tests. However, providing this type of feedback to drivers is expensive, which is why it occurs only when someone is initially learning to drive, or when they explicitly pay an expert to help improve their driving.

Due to great advances in mobile sensing technology, invehicle data recorders have emerged as a powerful tool to collect data on driving styles [36]. These platforms now 
allow a wide range of information to be recorded to understand interaction between the driver, the vehicle and the environment [2]. Various sensing platforms have been exploited, from the OBD2 and radar to cameras or even smartphones [22, 39], to collect information and understand driving style [2, 18, 24]. For example, Boyce and Geller used a smart car equipped with four cameras to monitor the driver and the roadway environment of the vehicle, and an on-line computer to measure information like vehicle velocity, and investigated relationships between age, personality and driving style [5]. Miyajima et al. analyzed drivers' car-following and pedal operation patterns for driver identification [24]. Also, the 100-Car Naturalistic Driving Study used an in-vehicle data recorder, an accelerometer, multiple cameras, and GPS to capture information on driver performance and behavior in the moments leading up to a crash [27]. Malta et al. used the multimodal signals of brake pedal use and driver speech in addition to vehicle speed to detect incidents from a realworld driving database [23].

Despite the promise of such an approach, these in-vehicle sensing platforms tend to be too expensive for the average consumer to own. To solve this problem, some researchers have turned to smartphones as a sensor platform, which are quite powerful, widely available, and are often already owned by drivers [19, 22, 39]. Smartphone owners now almost always have the device in their possession, and it can be an accurate proxy to collect a variety of information about users [13], even while driving. Johnson and Trivedi tried to characterize driving style based on smartphone-based sensors including an accelerometer, gyroscope, magnetometer, GPS, and camera [19], while You et al. used dual-cameras on smartphones to detect dangerous driving behavior [39]. Johnson and Trivedi used dynamic time warping to detect the type of maneuvering based on the movement of a smartphone mounted on a car in a controlled experiment, while You et al. have only presented the idea of using the dual-camera without any experimental validation. Despite their issues, these studies have shown that a lightweight smartphonebased sensing platform can collect useful information related to driving style.

\section{SENSOR-BASED EVALUATION OF DRIVING STYLE Lightweight Sensing Platform using Android Phones} Our In-Vehicle SmartPhone Sensing Platform (IV-SP2) is depicted in Figure 2. It differs from other sensing platforms that rely on powerful but heavyweight computers and expensive sensors [2, 14]. The core part of our system is the driver's own Android smartphone mounted on the front dashboard, which not only collects the location and movement of the vehicle but also accesses data from two additional sensors. Current Android smartphones are equipped with many sensing and communication capabilities, including dual cameras (front and back), microphones, GPS, a 3D accelerometer, a 3D gyroscope, a 3D magnetometer, a proximity sensor, an illumination sensor and a barometer, and $\mathrm{WiFi}, 3 \mathrm{G}$ and Bluetooth. They are powerful and portable enough for data collection in vehicles, and can even connect to other sensors through Bluetooth. Our system exploits most of these sensing modalities, but we do not include cameras and microphones due to privacy concerns.

In addition to the Android smartphone, we include two other modules: a Bluetooth-based on-board diagnostic (OBD2) reader and an inertial measurement unit (IMU) to support more diverse sensing modalities related to driving style (Figure 2). The OBD2 reader is connected to the OBD2 port of a vehicle and reads a variety of information from the vehicle, such as vehicle speed, engine RPM, and throttle position. It can stream the captured data via Bluetooth to the smartphone. The IMU (in our case, a YEI 3-Space Sensor ${ }^{\mathrm{TM}}$ Bluetooth), attached to the upper back of the steering wheel (toward the dashboard) without impacting its operation, and it captures the wheel movement with an accelerometer, gyroscope and compass sensors. It is also communicates via Bluetooth and can download data in real time. In this study, we collect all available variables every half second; Table 1 summarizes what our data sensing platform collects.

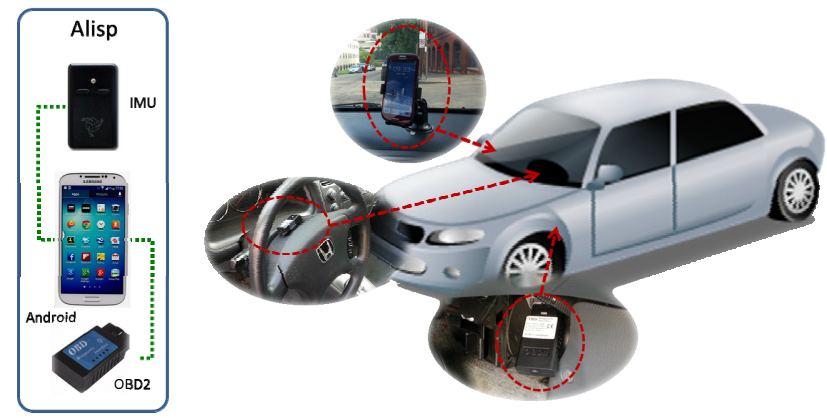

Figure 2. Overview of the Android-based in-vehicle sensing platform

\begin{tabular}{|l|l|l|}
\hline Source & Variables & Cost \\
\hline Android & $\begin{array}{l}\text { GPS (location, speed, bearing), 3D } \\
\text { acceleration, orientation, compass, } \\
\text { gyroscope, linear acceleration, gravity, } \\
\text { rotation vector, illumination, air pressure }\end{array}$ & $\begin{array}{l}\$ 0, \\
\text { already } \\
\text { purchased }\end{array}$ \\
\hline OBD2 & $\begin{array}{l}\text { Engine coolant temperature, engine load, } \\
\text { engine RPM, throttle position, speed }\end{array}$ & $\$ 25$ \\
\hline IMU & 3D acceleration, gyroscope, compass & $\$ 15-300$ \\
\hline
\end{tabular}

Table 1: Smartphone and sensor variables and costs

\section{Modeling Driving Style}

To use this sensed data to assess driving style, there are a number of steps to perform: preprocessing the data, extracting features, and creating a model of driving style.

\section{Preprocessing}

Ideally, smartphones and IMUs mounted in different vehicles would have the same orientation that would not change over time. In practice, however, vehicles usually have different shapes of steering wheels and dashboards, and users may mount them at an arbitrary orientation. In order to build a general model of driving styles across a population, we convert sensory signals to have a consistent orientation across all vehicles. A virtual reorientation method [25] is 
used to re-calibrate the $3 \mathrm{D}$ measurements, by repeatedly adjusting the $3 \mathrm{D}$ orientation of the smartphone and IMU with respect to their orientation when the vehicle stops moving. Also, information from the OBD2 is normalized per vehicle by simply subtracting baseline readings (car not in motion) from the in-motion readings.

\section{Feature Extraction}

To objectively characterize driving style based on sensor data, we define a number of driving features from the set of three sensors that are used in building a machine learning (ML)based model of driving style. Because naturalistic data collection does not force drivers to drive predefined routes, the features need to be independent of the context of any specific trip. For example, trips on highways and city roads are very different in their characteristics and a model built for one will not work well on the other. To address this, we instead define six specific driving situations, which we can use to compare a driver's driving style more independently of her own trips, including 1) START: for five seconds after a start, 2) STOP: for five seconds before a stop, 3) H-SPEED: while the vehicle is moving faster than $50 \mathrm{kph}$, 4) TURN: when the vehicle is turning (turn is detected based on z-axis acceleration of IMU) due to the road turning or a turn at an intersection, 5) B-TURN: for the five seconds just before the vehicle makes a turn, 6) A-TURN: for five seconds just after the vehicle makes a turn. In addition, we analyze certain driving features, such as maximum engine RPM and throttle position, across all driving trips.

To characterize one's driving style, we define the driving features based on the variables of the three sensors as shown in Table 2. The smartphone, mounted on the car, is used to detect speed (via GPS), speed change (acceleration), and the lateral and longitudinal acceleration of the car. The OBD2 reader, installed in the vehicle, records more fine-grained speed and also pulls some vehicle-specific data, such as engine RPM, accelerator (throttle) position, and the derivatives of those variables (note that we only use the three main variables (speed, engine RPM, and throttle position) out of a number of potential variables since they are commonly available from the vehicles of all drivers that participated in this study). From the IMU mounted on the steering wheel, we first detect turn events based on the change of z-axis acceleration, and identify three driving situations, including TURN, B-TURN, and A-TURN. IV-SP2 produces a sample composed of the driving features every half second.

\begin{tabular}{|l|l|}
\hline Source & Features \\
\hline Smartphone & $\begin{array}{l}\text { Max/avg/std of speed, speed change, } \\
\text { longitudinal/lateral acceleration }\end{array}$ \\
\hline OBD2 & $\begin{array}{l}\text { Max/avg/std of speed, speed change, engine } \\
\text { RPM, engine RPM change, throttle position, } \\
\text { throttle position change }\end{array}$ \\
\hline IMU & $\begin{array}{l}\text { Used to detect turn events based on its z-axis } \\
\text { acceleration change }\end{array}$ \\
\hline
\end{tabular}

Table 2: Features used in characterizing driving style
Aggressive Driving Style Ground Truth

Samples composed of the driving features defined in Table 2 are used to predict a driver's driving style by evaluating them for aggressiveness. To acquire the ground truth of whether a driver is aggressive, we use two methods: selfreports of accidents and ticket records, and a driving style questionnaire.

Measure 1: Driving violations

As one measure of aggressive driving style, a driver can be characterized by his record of driving violations. We characterize a driver as aggressive (violator) or not (nonviolator) using the number of accidents or tickets from the past 3 years. This time frame ensures that there is sufficient time for any accidents and tickets to accumulate, but is short enough so as not to mischaracterize drivers who have changed their driving patterns. Compared to questionnaire responses, this measure is more objective and more directly represents the risk of poor or aggressive driving style (unless a driver misrepresents his driving record). However, it lacks the nuance of questionnaires because it does not distinguish between the causes of the accidents or tickets.

Measure 2: Questionnaire responses

To complement the driving outcome record, we characterize drivers' driving style differently based on the responses to the DBQ [32] and additional aggressive driving related questions [7, 16]; drivers who score high are characterized as relatively aggressive and the others as relatively calm. Incorporating the latter questions that cover similar, but non-overlapping behaviors of aggressive violations and ordinary violations [21], allows a clearer division into aggressive and calm groups by allowing aggressive drivers to accrue more points. Aggressive violations are when a driver inconveniences or acts vengefully toward another driver, e.g., flashing his headlights for driving too slowly, which clearly falls under the common denotation for aggressive driving. Ordinary violations are when a driver violated highway laws and acted unsafely, but not aggressively towards another driver, e.g., driving too quickly or not coming to a complete stop, also refers to unsafe driving practices which can be improved through feedback. Aggressive violations and ordinary violations are given the same weight and summed as a measure of driving aggressiveness.

\section{Modeling Driving Style}

To create a machine learning (ML)-based model of driving style, we build a feature vector using the variables in Table 2 and use it to predict the target driving style. From the extracted driving features on the 6 driving situations of each trip of a driver, we first produce a feature vector (called trip-profile). A new feature vector (called driver-profile) for each driver is constructed by summarizing a number of his trip-profiles obtained over 3 weeks. From the driverprofiles and ground truth of drivers, the ML-based driver model is built to evaluate a driver's aggressiveness. Given an input driver-profile feature vector, the driver model 
evaluates a driver's driving style: violator, non-violator, aggressive, or calm, from our two measures.

Before creating the model, all the continuous driving features are discretized into five states v very low, low, medium, high, very high $\}$ where the range of values in each state is chosen to make the number of samples equivalent across the states. Then to create the driver model, we select informative driving features based on their information gain and apply a probabilistic technique, a naive Bayes classifier (NB), to model the relationship between driving style and the selected driving features. NB is a simple probabilistic model based on Bayes' theorem and independence assumptions of features. Despite its use of over-simplified assumptions, it often exhibits good performance on many real-world problems and the resulting model can be easily interpreted and understood.

In modeling driving style from our driving features, one goal is to assess driving style with a set of affordable sensors. To this end, we create three models to predict driving styles using the driver-profiles.

- Model 1, Smartphone Only: This model does not require any installation of external sensors. It will capture only the general movement of a vehicle.

- Model 2, Smartphone and OBD2: This model only requires the simple and quick installation of an OBD2 reader. The OBD2 reader costs about $\$ 25$. This model provides more accurate and detailed information about vehicle operation.

- Model 3, Smartphone, OBD2, and IMU: The sensors for this model cost more than the other models because of the IMU. However, a Nintendo Wii remote is a potential replacement for our IMU, and costs only $\$ 15$ and provides 3D acceleration. (Note that our choice of IMU instead of the Wii remote is due to the usability of its programmatic interface and usability in a car.) This model additionally incorporates information about steering wheel control, when mounted on the steering wheel.

\section{METHOD}

We recruited 22 licensed drivers (11 males and 11 females) from a mid-sized city in the United States, ranging in age from 21 to 34 years $(M=26)$. A prescreening questionnaire was used to recruit a number of people in our target population: drivers who owned and used an Android smartphone and drove at least several hours per week. Participants were asked to drive their own vehicles, which we equipped with our sensing platform, and to use their usual daily driving routes plus a predesigned 10-minute driving course (once per week) to verify the proper operation of the sensing platform. As described earlier, all sensor data are collected and stored on their smartphone.

During their first visit to our lab, we installed an IMU and an OBD2 reader in their vehicles and a logging application on their smartphones, and provided brief instructions on how to use the system. Then, they were asked to list their driving violations for the past 3 years, and complete our driving behavior questionnaire. For each question, participants ranked the frequency of a driving behavior, on a six-point Likert-scale (ranging from $0=$ never, $5=$ nearly all the time). Participants were required to visit our lab each week for data downloading, and were compensated with $\$ 60$ for completing 3 weeks of data collection. A total of 542 hours of driving data were collected from 1,017 trips.

\section{RESULT \& ANALYSIS}

\section{Questionnaire Analysis}

According to the self-reports of accidents and speed violations, we divided participants into two groups: violator and non-violator (we will call this group division violationclass). As an additional measure of aggressive driving style based on the questionnaires, we also divided our participants into two groups: relatively aggressive and relatively calm. (Note that the term relatively indicates that our participants cover a subpopulation of drivers, the division is measured by looking at the relative interquartile ranges for aggressive versus calm drivers, and we will call this group division questionnaire-class and use aggressive and calm without mentioning the term relatively in the rest of the paper).

Figure 3 shows the basic distribution of our population in terms of their violation-class and questionnaire-class. Out of the 22 participants, 12 were characterized as violators; these drivers had an average of 2 accidents and tickets. 11 of the participants were characterized as aggressive, where they had an average score of $28.1 \%(\sigma=0.05)$ from the questionnaire, compared to calm drivers' scoring $16 \%$ $(\sigma=0.05)$. While there was a significant participant overlap between violators and aggressive drivers (10 participants), the participants' questionnaire scores were not always correlated with their accident/ticket record. The correlation across all drivers is 0.26 .
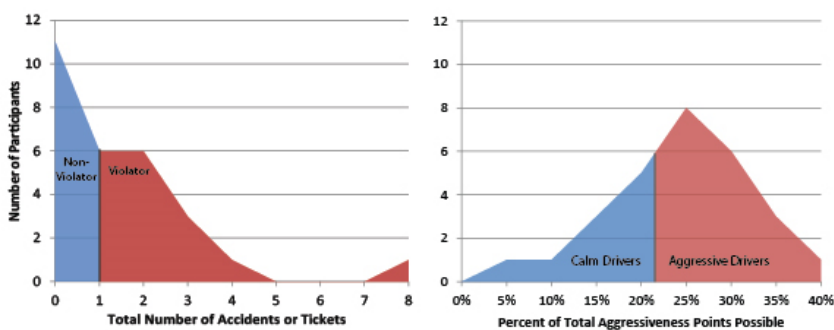

Figure 3. Distribution of Accident \& Ticket Record and Questionnaire Responses

\section{Sensor-based Driving Style Analysis}

In order to have an understanding of the relationship between the sensor data and driving style, we first compared the driving features from drivers (10) who were classified as aggressive violators (i.e., those who were classified as both aggressive and violators) versus calm non-violators (6). This allows the data to more clearly define aggressive driving style. The remaining 6 
participants who were either aggressive non-violators or calm violators will be discussed later in this paper.

Classifying the sensor-based driving feature data as coming from aggressive violators or calm non-violators yields two findings. First, in line with intuition, the average aggressive-violator generally experiences higher g-forces (forces the body is subjected to during acceleration). Second, aggressive violators have larger within-person and between-people variation, which signifies that their individual driving style is more inconsistent.

\section{Aggressive Drivers and Violators Have Higher G-Forces} Contrary to our intuition, both aggressive violators and calm non-violators have similar maximum speeds, as shown in Figure 4a; for naturalistic driving data, speed is not a good way to distinguish aggressive and calm drivers. However, its derivative, acceleration, proves to be. Aggressive violators have higher maximum acceleration and deceleration (derived from speed) throughout their trips in general and also in specific situations, such as during a turn (Figure 4b), when driving at high speed (Figure 4c), accelerating from a stopped position, and decelerating to stop their vehicles.
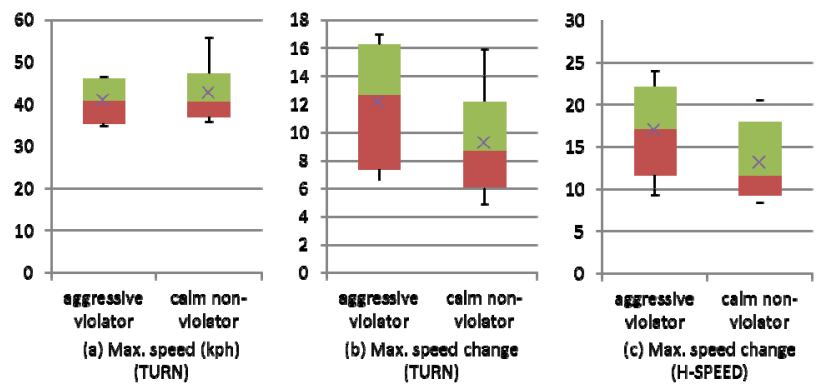

Figure 4. Driving feature distribution on aggressive driving style

Furthermore as shown in Figure 5, in the 5 seconds after an aggressive violator begins moving the car, the car has a higher maximum speed and engine RPM, but shows the same average speed and engine RPM as for a calm nonviolator. Figure 5 implies that aggressive violators tend to wait longer to make a change to their speed, and then make a more forceful change. In contrast calm non-violators may respond sooner and make lower magnitude changes.

Many driving features extracted from our sensing platform more directly support the fact that aggressive violators have higher g-forces for both acceleration and deceleration, such as higher smartphone acceleration in the vehicle's longitudinal (y-axis) and lateral (x-axis) directions. For instance, the acceleration of the smartphone shows aggressive violators experience higher g-forces than calm non-violators when travelling at high speeds (>50 kph), which means the higher g-forces they experience are not limited to starting and stopping the vehicle, but also to highway travel (e.g., a quick deceleration from $100 \mathrm{kph}$ to $60 \mathrm{kph}$ or weaving between lanes in high speed traffic with frequent lane changes). More concretely, when an aggressive violator accelerates from a stop, she has a maximum lateral acceleration of $.38 \mathrm{~g}$ versus $.35 \mathrm{~g}$ for calm non-violators, and a maximum longitudinal acceleration of $.39 \mathrm{~g}$ versus $.31 \mathrm{~g}$ for calm non-violators. These are the same g-forces that put strain on the vehicle and can be felt by passengers in the car.
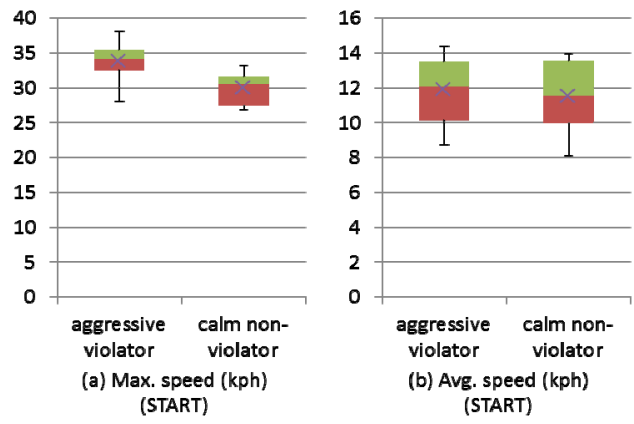

Figure 5. Speed comparison on START
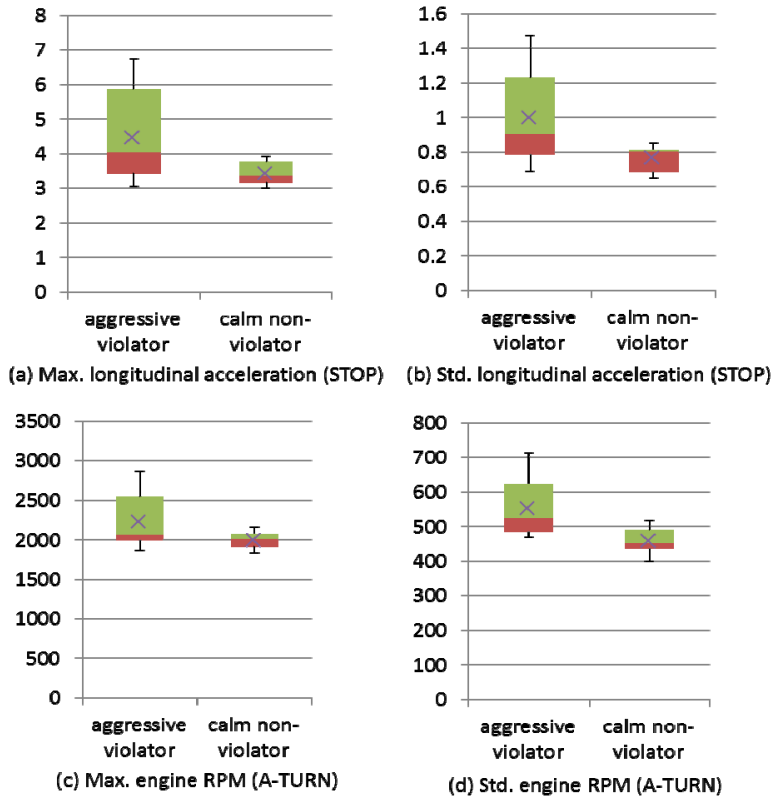

Figure 6. Consistency analysis of drivers

Aggressive Drivers and Violators Behave Less Consistently Aggressive violators, in contrast to calm non-violators, have an inconsistent driving style, as evidenced by several driving features, such as the change of speed and the selection of engine RPMs or throttle positions in our driving situations, extracted from the sensor data. First, aggressive violators have lower levels of group consistency in general, meaning that drivers who are classified as aggressive by the questionnaire have a greater variation for many metrics than do drivers who are classified as calm non-violators. This is measured by looking at the relative interquartile ranges (IQR) for aggressive violators versus calm non-violators. Aggressive violators have a broader distribution of maximum longitudinal acceleration for the 5 seconds before the vehicle comes to a stop as shown in Figure 6. There is a similar result for the maximum engine 
RPM for the 5 seconds after the vehicle makes a turn. This finding implies that one potential application of this research could be to identify a particular driver behavior as being aggressive or calm, and then provide feedback to the driver on the specific behavior that needs to be corrected. This finding also indicates that an aggressive violator driving style is characterized by multiple driving features. Because some aggressive violators have a lower maximum RPM and longitudinal acceleration than some calm nonviolators, we must use a sophisticated model that incorporates a number of driving features.

Second, aggressive violators have lower levels of individual consistency in most of our driving features (as calculated from their average standard deviations over all of their trips), meaning that an average aggressive driver will be on the lower end of a variable for some trips, and on the higher end of that same spectrum on other trips. In contrast, an average calm non-violator will behave more similarly from trip to trip. For instance, as shown in Figure 6b, calm non-violators have a lower standard deviation of longitudinal g-forces when coming to a stop. This finding also holds when looking just at high-speed driving, after starting the car, and during a turn. Especially when drivers are travelling faster than $50 \mathrm{kph}$, the maximum, average and standard deviation of the lateral g-forces (measured by the $\mathrm{x}$-axis acceleration of the smartphone) of aggressive violators is larger than those of calm non-violators. This indicates that an average aggressive driver subjects the car and its passengers to a larger variety of g-forces than do calm non-violators, who are more consistent.

\section{Building a Machine Learning Model}

From our feature analysis, we created two driver models that predict whether a driver has an aggressive driving style or not, one determined by the record of accidents and speeding tickets (violator-class), and another determined by the questionnaire responses (questionnaire-class). To model the relationship of driving features from the three data sources (smartphone, OBD2, and IMU) with each class, we perform feature selection using information gain and use a naïve Bayes classifier with 5-bin discretization, and we conduct leave-one subject-out cross validation for performance evaluation. Figure 7 shows the result.

\section{IV-SP2-Based Model Accuracy}

The average model accuracy with all three sensors was $90.5 \%$ ( 2 false negatives) when predicting the violationclass and $81 \%$ ( 2 false negatives $\& 2$ false positives) when predicting the questionnaire-class. These are particularly high accuracies, compared with the baseline model (ZeroR, which always predicts the most frequent classification): $57 \%$ and $52.4 \%$ for violation-class and questionnaire-class, respectively. We also found that our driver model performed relatively well without using all the sensors in the IV-SP2 system. More specifically, when modeling the violation-class with just the smartphone and the OBD2 (without considering turn situations (B-TURN, TURN, and
A-TURN, identified by steering wheel information, using the IMU), we achieved $81 \%$ accuracy. With only the smartphone (without utilizing car information, such as RPM and throttle position), we achieved $66.7 \%$ accuracy. Similarly, when predicting the questionnaire-class, the model is $76.2 \%$ accurate with the smartphone and OBD2 only, and $66.7 \%$ with just the smartphone.

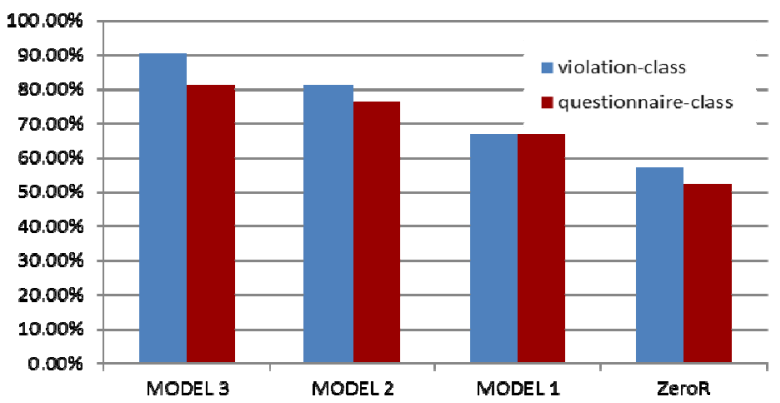

Figure 7. Prediction of aggressive driving style. Model 1 uses smartphone data only, Model 2 uses smartphone and OBD2 data, and Model 3 uses smartphone, OBD2 and IMU data.

Participant S18, a violator, provides an example of a misclassification when fewer sensors are used to predict driving style. When using the 3-sensor IV-SP2 system, S18 is classified correctly as a violator. However, with only the smartphone and OBD2 reader, he is classified incorrectly as a non-violator. This occurred because his general driving style is mild but he is very aggressive in his turning behavior, e.g., he often accelerates more than an average violator while turning. As this behavior occurs frequently, the 3-sensor model correctly characterizes him as a violator. With our sensor-based driving feature data, we achieved higher model accuracy when predicting the violation-class as opposed to the questionnaire-class $(90.5 \%$ vs. $81 \%)$.

\section{DISCUSSION}

In the discussion section, we will address issues on the applicability of our IV-SP2 and driver models in practice, limitations of using the driving questionnaire, and difficulty of conducting naturalistic driving data collection.

\section{Applying IV-SP2 to Real-World Driving in Practice}

Using our driving models, we can assess driving style at three different levels. First, our sensing platform can collect one's naturalistic driving data and, based on all trips taken, let the driver know whether she has an aggressive driving style which may lead to an increased risk of accidents. This level of assessment can be performed periodically to coach drivers "at risk" for aggressive driving styles, e.g., retraining drivers who have at-fault accidents or who have received a number of speed tickets over some time period, or to monitor for a decrease in driver performance due to aging or cognitive disorder [3]. Second, the driver models can evaluate one's driving style on a particular trip. Due to a number of internal and external factors, e.g., stress [4], heavy traffic and road construction, aggressiveness can increase, which may increase the risk of an accident. 
Objective feedback about an elevated risk may encourage the driver to correct his or her driving. Figure 8 shows the evaluations of driving style for each trip for each participant. Drivers characterized as being aggressive have more aggressive trips in general, but are not aggressive on all trips. Likewise, a non-aggressive driver sometimes drives aggressively.

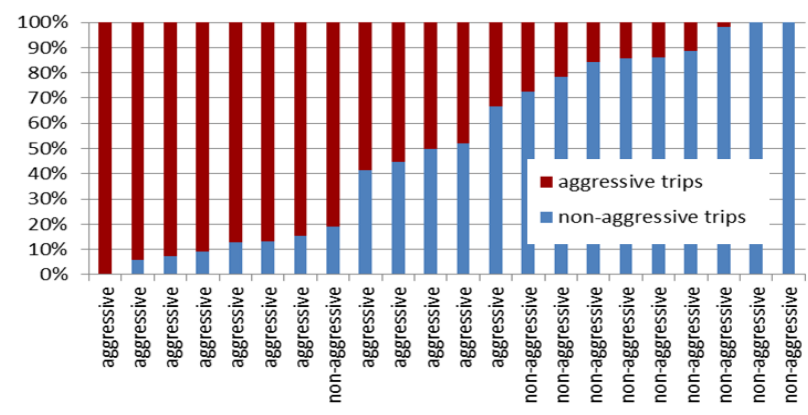

Figure 8. Assessment of individual trips

Third, our driver models can detect aggressive driving in specific situations and provide feedback on driving behaviors. A driver who has an aggressive driving style could get specific advice on how to change her driving style, e.g., keeping a constant engine RPM when driving at a high speed instead of frequently changing speed. Also, if a driver turns very aggressively, the system can coach him to make a better/safer turn. By expanding this to a wider variety of driving situations, it is also possible to provide both more specific assessments and feedback.

Still, the way that characterizes driving styles has to be diversified to present a broader spectrum for understanding aggressive driving styles. For example, considering driving style as a continuous variable will better reflect reality and improve the capability of our system in practice.

\section{Limitations of Questionnaire-Based Classification and its Impact on Our Model}

While questionnaires can provide a cost effective and fast method for assessing driving style, they suffer from a few weaknesses when they are used as an initial starting point to build a driver model with machine learning.

Research suggests that behavioral questionnaires yield responses that are loosely correlated with real world poor driving outcomes [38]. Assuming that these responses are less accurate than self-reports of one's accident and ticket record, the driver model based on the questionnaire-class may have inherited the same limitation. Three findings point us in this direction.

First, one effect of this can be seen by cross referencing the responses from the questionnaire and the self-reported accident and ticket record. If the questionnaire responses were perfectly correlated with real-world poor driving outcomes, we would expect all aggressive drivers to be classified as violators, and calm drivers as non-violators. In fact, for 6 participants (24\%), this relationship does not hold. These drivers could be referred to as "lucky" (2 participants classified as aggressive, but also as a nonviolator) or "unlucky" (4 participants classified as calm, but also as a violator). Especially, being referred to as unlucky could be because they have been really unlucky, or because their poor driving is due to errors and lapses (which are not captured in our questionnaire), or because their responses on the questionnaires are not accurate.

Second, for the majority of driving features we examined, the distinction between violators and non-violators was much clearer than the distinction between aggressive and calm drivers. However, when comparing aggressive versus calm drivers, we cannot so easily find a distinction: the averages and IQRs are much more similar. Thus, when we group participants according to their more objective accident and ticket record, the distinction between groups is much clearer than when- we group them solely by their self-reported driving behavior.

Third, the sensor-based driver model did not predict the questionnaire-class as accurately as it did the violationclass $(81 \%$ vs $90.5 \%)$. The misclassifications in the questionnaire-class model occurred for 3 drivers, which we believe occurred because these drivers were either lucky or unlucky. The misclassifications of the model may be due to the limitation of the more subjective questionnaire approach. Accidents and tickets are objective records of driving style or performance, as are the data from the IVSP2 system. Future work in this line of research should investigate a more accurate ground truth that includes driver perception and verified accident and ticket data. In addition, further augmenting with range sensors and cameras will provide higher-fidelity ground truth and a validation study using multiple methods would greatly contribute to the field.

\section{Difficulties of Naturalistic Data Collection}

Recording naturalistic data provides a more objective view of driver behavior, but it still presents challenges with data processing, some of which were easily surmountable, while others require more work.

For example, because people generally drive on the same roads over a short period of time, the variety of road type data for each participant is not the same. While we attempted to control this by collecting data over 3 weeks, some calm drivers spent much of their time traveling faster on the highways, while some aggressive drivers spent a lot of their driving time sitting at red lights on city streets. Without controlling where our participants drove, as has been done in other studies, we could not use those variables that would be affected by this bias (e.g., speed), when averaging across all driving behaviors. For the variables, we were able to find more distinction between aggressive and calm drivers only in specific driving situations (e.g., high speed data, or at vehicle starts and stops).

Also, the fact that each participant is driving his or her own car and using his or her own smartphone creates an additional source of bias in the data that is difficult to 
control for. Some variables coming from the car, such as the engine load, are very sensitive to the type of car and transmission in the car. For these variables, we created new data features based on the difference and performed basic calibrations such as normalization and subtracting the baseline. Still, these calibrations are not ideal; further work is required to improve the quality of the sensor data and more reliably and accurately extract values of the variables.

Third, one limitation of the ML-based model which only uses the smartphone is that it uses the GPS data to determine speed. GPS sampling rates are not high enough to reliably determine speed with enough nuance; sampling rates were closer to $1 / 2 \mathrm{~Hz}$ as opposed to $4-5 \mathrm{~Hz}$ for the other sensors. Furthermore, the GPS data becomes inaccurate around large buildings and in tunnels; for this reason, even though the phone-only model achieves good accuracy, adding an OBD2 reader provides an alternate source of speed information.

Finally, the IV-SP2 is limited in its ability to detect the driver's road context, since it is optimized to capture information on how the driver is controlling the car. We suspect that variables such as the weather and traffic or even the body position of the driver, could improve the classification accuracy by allowing the model to understand some intentionality behind behaviors. As our system solely focuses on the driver's behavior, additional contextual information could improve the predictability of our model by allowing a more nuanced look at the causes and correlates of particular aggressive driving behaviors.

\section{CONCLUSION}

In this paper, we introduced the In-Vehicle Smartphonebased Sensing Platform (IV-SP2), which has the potential to provide real-time feedback to drivers using their own Android smartphone with relatively cheap sensors. With this sensing platform, we collected a variety of information from drivers' naturalistic driving, e.g., speed, acceleration, deceleration, engine RPM, throttle position, and steering wheel movement, and built driver models to predict aggressive driving style. With naturalistic driving data collected from 22 drivers, we could identify aggressive driving (in an accuracy of $90 \%$ for violation-class and $81 \%$ for questionnaire-class). In the future, we aim to enhance IV-SP2 by introducing new driving situations and driving features to assess more diverse driving styles, and will develop and deploy an end-user application that provides three levels of feedback to improve driver's driving style.

\section{REFERENCES}

1. Aggressive driving: research update, American Automobile Assoc. Foundation for Traffic Safety, (2009).

2. Amditis, A., Pagle, K., Joshi, S., and Bekiaris, E. Driver-vehicle-environment monitoring for on-board driver support systems: lessons learned from design and implementation. Applied Ergonomics 41, 2 (2010), 225235.

3. Barkley, R.A. and Cox, C. A review of driving risks and impairments associated with attentiondeficit/hyperactivity disorder and the effects of stimulant medication on driving performance. $J$. of Safety Research 38, 1 (2007), 113-128.

4. Berdoulat, E., Vavassori, D., and Sastre, M. Driving anger, emotional and instrumental aggressiveness, and impulsiveness in the prediction of aggressive and transgressive driving. Accident Analysis and Prevention 50, (2013), 758-767.

5. Boyce, T.E. and Geller, E.S. An instrumented vehicle assessment of problem behavior and driving style: Do younger males really take more risks? Accident Analysis and Prevention 34, 1 (2002), 51-64.

6. Cambridge Systematics. 2011. Crashes vs Congestion: What's the Cost to Society? American Automobile Association, (November 2011). Retrieved Sept. 5, 2013,

7. Chilaoutakis, J.E., Demakakos, P., Tzamalouka, G., Bakou, V., Koumaki, M., and Darviri, C. Aggressive behavior while driving as predictor of self-reported car crashes. J. of Safety Research 33, 4 (2002), 431-443.

8. Coughlin, J.F., Reimer, B., and Mehler, B. Monitoring, managing, and motivating driver safety and well-being. Pervasive Computing IEEE 10, 3 (2011), 14-21.

9. Countermeasures that work: A highway safety countermeasure guide for state highway safety offices. National Highway Traffic Safety Administration Fourth Edition. (2009).

10.Dahlen, E.R., Edwards, B.D., Tubre, T., Zyphur, M.J., and Warren, C.R. Taking a look behind the wheel: An investigation into the personality predictors of aggressive driving. Accident Analysis \& Prevention 45, (2012), 1-9.

11. Deffenbacher, J.L., Oetting, E.R., and Lynch, R.S. Development of a driving anger scale. Psychological Reports 74, 1 (1994), 83-91.

12.Deffenbacher, J.L., Oetting, E.R., and Swaim, R.C. The Driving Anger Expression Inventory: A measure of how people express their anger on the road. Behavior Research and Therapy 40, 6 (2002), 7-737.

13. Dey, A.K., Wac, K., Ferreira, D., Tassini, K., Hong, J.H., and Ramos, J. Getting closer: an empirical investigation of the proximity of user to their smart phones. Ubicomp'11, (2011), 163-172.

14. Espinosa, F., Jimenez, J.A., Santiso, E., Gardel, A., Perez, D., Casanova, J. and Santos, C. Design and implementation of a portable electronic system for vehicle-driver-route activity measurement. Measurement 44, 2 (2011), 326-337. 
15. Evans, L. 1991. Traffic Safety and the Driver. Van Nostrand, New York, NY.

16. Hennessey, D. and Weisenthal, D.L. Traffic congestion, driver stress, and driver aggression. Aggressive Behavior 25, 6 (1999). 409-423

17.Houston, J.M., Farris, P.B., and Norman, M. The aggressive driving behavior scale: developing a selfreport measure of unsafe driving practices. North American J. of Psychology 5, 2 (2003), 269-278.

18. Jensen, M., Wagner, J., and Alexander, K. Analysis of in-vehicle driver behaviour data for improved safety. Int. J. of Vehicle Safety 5, 3 (2011), 197-212.

19. Johnson, D.A. and Trivedi, M.M. Driving style recognition using a smartphone as a sensor platform. In Proc. $11^{\text {th }}$ Int. Conf. Mobile and Ubiquitous Multimedia, (2011), 1609-1615.

20. Kee, S., Shamsul, B, and Goh, Y Differences of drivers' driving performance in simulated driving. Research $J$. of Applied Sciences 4, 6 (2009), 230-237.

21.Lawton, R., Parker, D., Manstead, A.S.R., and Stradling, S.G. The role of affect in predicting social behaviors: the case of road traffic violations. J. of Applied Social Psychology 27, 14 (1997), 1258-1276.

22.Li, K., Lu, M., Lu, F., Lv, Q., Shang, L., and Maksimovic, D. Personalized driving behavior monitoring and analysis and emerging hybrid vehicles. Pervasive (LNCS 7319), (2012), 1-19.

23. Malta, L., Miyajima, C. and Takeda, K. A study of driver behavior under potential threats in vehicle traffic. IEEE Transactions on Intelligent Transportation Systems 10, 2 (2009), 201-210.

24. Miyajima, C., Nishiwaki, Y., Ozawa, K., Wakita, T., Itou, K.,Takeda, K., and Itakura, F. Driver modeling based on driving behavior and its evaluation in driver identification. Proc. of the IEEE 95, 2 (2007), 427-437.

25. Mohan, P., PadManabhan, V.N., and Ramjee, R. Nericell: rich monitoring of road and traffic conditions using mobile smartphones. SenSys'08, (2008), 323.

26. Mynttinen, S., Sundstrom, A., Vissers, J. Koivukoski, M., Hakuli, K., and Keskinen, E. Self-assessed driver competence among novice drivers - a comparison of driving test candidate assessments and examiner assessments in a Dutch and Finnish sample. J. of Safety Research 40, 4 (2009), 301-309.

27. Neale, V.L., Dingus, T.A., Klauer, S., Sudweeks, J. and Goodman, M. An overview of the 100-car naturalistic study and findings. ESV Paper 05-0400, (2005).
28. Nichols, J. New opportunities: Can behavior analysis help with traffic safety? Panel discussion (panel session). 24th Annual Convention of the Association for Behavior Analysis, (1998).

29. Owsley, C. Driving mobility, older adults, and quality of life. Gerontechnology 1, 4 (2002), 220-230.

30.Özkan, T., Lajunen, T., Chliaoutakis, J.E., Parker, D., and Summala, H. Cross-cultural differences in driving behaviours: A comparison of six countries.

Transportation Research Part F: Traffic Psychology and Behaviour 9, 3 (2006), 227-242.

31.Paefgen, J., Kehr, F., Zhi, Y., and Michaelles, F. Driving behavior analysis with smartphones: insights from a controlled field study. In Proc. $11^{\text {th }}$ Int. Conf. Mobile and Ubiquitous Multimedia, (2012), 36.

32. Reason, J., Manstead, A., Stradling, S., Baxter, J., and Campbell, K. Errors and violations on the roads: a real distinction? Ergonomics 33, 10-11 (1990), 1315-1332.

33. Shinar, D. and Compton, R. Aggressive driving: an observational study of driver, vehicle, and situational variables, Accident Analysis and Prevention 36, 3 (2004), 429-437.

34. Sundström, A. Self-assessment of driving skill - A review from a measurement perspective. Transportation Research Part F, 11 (2008), 1-9.

35. Tasca, L. A review of the literature on aggressive driving research. Aggressive Driving Issues Conf., (2000).

36. Toledo, T., Musicant, O., Lotan, T. In-vehicle data recorders for monitoring and feedback on drivers' behavior. Transportation Research Part C 16, 3 (2008), 320-331.

37. Wiesenthal, D.L., Hennessy, D., and Gibson, P.M. The Driving Vengeance Questionnaire (DVQ): The development of a scale to measure deviant drivers' attitudes. Violence and Victims 15, 2 (2000), 115-136.

38. Winter, J. and Dodou, D. The driver behavior questionnaire as a predictor of accidents: a metaanalysis. J. of Safety Research 41, 6 (2010), 463-470.

39. You, C.-W., Montes-de-Oca, M., Bao, T.J., Lane, N.D., Lu H., Cardone, G., Torresani, L., and Campbell, A.T. CarSafe: a driver safety app that detects dangerous driving behavior using dual-cameras on smartphones. Ubicomp'12, (2012), 671-672.

40.Zhang, Y., Lin, W.C., and Chin, Y.-K. A patternrecognition approach for driving skill characterization. IEEE Trans. on Intelligent Transportation Systems 11, 4 (2010), 905-916. 